[Internet]. Washington, D.C., USA: PAHO; 2011 [actualizado el 11 de noviembre; citado el 3 de mayo de 2017]. Disponible en: http://www.paho.org/hq/index.php?option=com_content\&vie $\mathrm{w}=$ article\&id $=6152 \% 3 \mathrm{~A} 2011$-countries-americas-taking-actionreduce-salt-consumption-save-lives\&Itemid=1926\&lang=en.

6. Population health Division. Technical guidance on nutrition labelling. In: Department of health. London, UK: UK goverment; 2017.p. 22.

Correspondencia: Diego Azañedo

Dirección: Jr. Tumbes 247, Casco urbano, Chimbote, Ancash, 02804, Perú. Teléfono: (43) 947121972

Correo electrónico:dazanedov@uladech.edu.pe

\section{PRODUCCIÓN CIENTÍFICA EN BASE A CINCO ENCUESTAS NACIONALES DE PERÚ}

\section{SCIENTIFIC PRODUCTION BASED ON FIVE NATIONAL SURVEYS IN PERU}

\author{
Juan C. Ruiz-Maza ${ }^{1, a}$, Armando M. Pezo-Pezo, a, \\ Renato A. Soto-Azpilcueta ${ }^{1, a}$
}

Sr. Editor. El Instituto Nacional de Estadística e Informática (INEI) ejecuta encuestas nacionales que recogen información sobre temas específicos como la salud reproductiva materno infantil y prevalencia de enfermedades transmisibles, las relaciones sociales, la calidad de vida de los hogares, entre otros. Cada encuesta tiene una periodicidad determinada y una metodología que busca la representatividad regional o nacional de sus resultados, destacando la Encuesta Demográfica y de Salud Familiar (ENDES) como la de mayor antigüedad (con datos desde 1986) ${ }^{(1)}$.

Los datos (informes y microdatos) de estas encuestas son de acceso libre desde el 2000 y se encuentran disponibles en la página web del INEI (http://iinei.inei.gob.pe/microdatos/). En este sentido, son una alternativa económicamente rentable para que nuevos investigadores desarrollen contenido científico de calidad y que pueda servir como evidencia en la toma de decisiones para la salud pública del país ${ }^{(2-4)}$. Por ello, surge el interés por conocer el uso de las encuestas nacionales peruanas como fuente generadora de investigaciones a lo largo del tiempo.

\footnotetext{
Sociedad Científica de San Fernando, Universidad Nacional Mayor de San Marcos. Lima, Perú.

a Estudiante de medicina

Recibido:06/11/2017 Aprobado:30/11/2017 En línea:05/04/2018
}

Citar como: Ruiz-Maza JC, Pezo-Pezo AM, Soto-Azpilcueta RA. Producción científica en base a cinco encuestas nacionales de Perú. Rev Peru Med Exp Salud Publica. 2018;35(1): 166-7.doi: 10.17843/rpmesp.2018.351.3554.
Se realizó un estudio bibliométrico sobre la producción científica, durante el periodo 1986-2017, en base a la utilización de datos de cinco encuestas desarrolladas en Perú: Encuesta Demográfica y de Salud Familiar (ENDES), Encuesta Nacional de Hogares (ENAHO), Encuesta Nacional de Relaciones Sociales (ENARES), Encuesta Nacional Especializada de Discapacitados (ENEDIS) y Encuesta Nacional de Satisfacción de Usuarios en Salud (ENSUSALUD).

La búsqueda se realizó en Scopus, Medline y SciELO, en la estrategia de búsqueda se utilizó las siguientes palabras clave: "encuesta nacional", "National survey", "DHS", "Encuesta Demográfica y de Salud Familiar","Demographich and health survey", "Demographic and Family Health Survey", "ENDES", "Encuesta Nacional de Hogares" ,"National Household Survey","ENAHO”, "Encuesta Nacional de Relaciones Sociales","national survey on social relations","ENARES", "Encuesta Nacional de Satisfacción de Usuarios en Salud", "National Survey of Satisfaction of Users in Health" "ENSUSALUD", "Encuesta Nacional Especializada de Discapacitados", "Disability National Survey ","ENEDIS”.

Se incluyeron los siguientes tipos de estudios: artículos originales, originales breves, revisiones y cartas al editor. La búsqueda se realizó del 1 al 7 de setiembre de 2017 , fueron categorizados por año de publicación y revista. Los datos fueron tabulados y analizados en Excel 2007.

Ingresaron al estudio 73 publicaciones de los cuales el $93,1 \%$ fueron artículos originales, el $84,9 \%$ fueron publicadas desde el 2000 y el 74,0\% utilizaron los datos de la ENDES. Al revisar la indización de las revistas donde se realizó la publicación se encontró que el 43,8\% estaban en Scopus y Medline. Además, la mayoría de publicaciones se realizó en la Revista Peruana de Medicina Experimental y Salud Pública (17,8\%) (Tabla 1).

Nuestros resultados demuestran el uso de los datos de las encuestas nacionales, principalmente ENDES o ENAHO, en el desarrollo de publicaciones en revistas nacionales e internacionales indizadas en bases de datos con elevados criterios de rigurosidad y calidad ${ }^{(4,5)}$.

La disponibilidad virtual de los datos de encuestas nacionales como la ENDES influye en el nivel de producción científica a través del tiempo ${ }^{(3)}$. Esto también se observa en nuestro estudio y explica la diferencia del nivel de productividad científica de encuestas con mayor antigüedad como ENDES y ENAHO (disponibles desde el 2000) en comparación a las encuestas más recientes como ENARES, ENSUSALUD y ENEDIS (disponibles desde el 2013).

Asimismo, el $40,1 \%$ de estas investigaciones fueron publicadas en solo cinco revistas, dato similar al reportado en estudios previos ${ }^{(3)}$. La elección de una revista esta 
relacionada con su indización, su eje temático, el idioma y los resultados del estudio. No se realizó el análisis de la temática de los artículos, pero es probable que estos tengan relevancia en salud pública, lo que explicaría la predominancia de la RPMESP (revista indizada en SciELO, Medline y Scopus). Se destaca la presencia de publicaciones en revistas indizadas de mayor impacto como la Revista Panamericana de Salud Pública y PloS ONE (4).

Un punto que no se ha podido explorar detalladamente es el impacto que tiene el uso de las encuestas nacionales en el desarrollo de tesis de posgrado. Principalmente porque muchas de las tesis no son referidas como tales al momento de la publicación; pese a ello, se han encontrado casos en los que si se ha dado dicha referencia ${ }^{(5)}$. Las encuestas nacionales al ser una fuente de datos secundarios,

Tabla 1. Características de la producción científica en base a cinco encuestas nacionales de Perú, 1986-2017.

\begin{tabular}{|c|c|c|}
\hline Características & $\begin{array}{l}\text { Número de } \\
\text { publicaciones } \\
(n=73)\end{array}$ & Porcentaje \\
\hline \multicolumn{3}{|l|}{ Tipo de artículo } \\
\hline Artículo original & 68 & 93,2 \\
\hline Artículo de revisión & 2 & 2,7 \\
\hline Otros & 3 & 4,1 \\
\hline \multicolumn{3}{|l|}{ Año de publicación } \\
\hline Antes del 2000 & 11 & 15,1 \\
\hline De 2000 al 2004 & 9 & 12,3 \\
\hline De 2005 al 2009 & 8 & 11,0 \\
\hline De 2010 a 2014 & 21 & 28,8 \\
\hline Desde el 2015 & 24 & 32,8 \\
\hline \multicolumn{3}{|l|}{ Encuesta utilizada } \\
\hline ENDES & 54 & 74,0 \\
\hline ENAHO & 10 & 13,7 \\
\hline ENDES y ENAHO & 3 & 4,1 \\
\hline Otros & 6 & 8,2 \\
\hline \multicolumn{3}{|l|}{ Revista donde se publicó } \\
\hline RPMESP & 13 & 17,8 \\
\hline $\begin{array}{l}\text { Revista Panamericana de } \\
\text { Salud Pública }\end{array}$ & 7 & 9,6 \\
\hline PLoS ONE & 4 & 5,5 \\
\hline Studies in Family Planning & 4 & 5,5 \\
\hline Notas de Población & 2 & 2,7 \\
\hline Otros & 43 & 58,9 \\
\hline \multicolumn{3}{|l|}{ Indización } \\
\hline Solo Scopus & 11 & 15,1 \\
\hline Solo SciELO & 5 & 6,8 \\
\hline Solo Medline & 1 & 1,4 \\
\hline Scopus y Medline & 32 & 43,8 \\
\hline Scopus y SciELO & 2 & 2,7 \\
\hline Scopus, Medline y SciELO & 22 & 30,2 \\
\hline
\end{tabular}

ENAHO: Encuesta Nacional de Hogares, ENDES: Encuesta Demográfica y de Salud Familiar, RPMESP: Revista Peruana de Medicina Experimental y Salud Pública minimizan el tiempo que el tesista debe invertir en la recolección de datos (y toda la logística que esto implica), permitiéndole reducir considerablemente el tiempo que implica el desarrollo de su tesis y, por ende, el tiempo que tarda en la obtención del grado académico.

La principal limitación del estudio es una posible subestimación de los resultados al no tomar en consideración otras bases de datos de menor rigurosidad científica como Lilacs o Lipecs; así como la existencia de artículos publicados en 2017 en una fecha posterior a la realización de la búsqueda. Además, este estudio no determinó el autor con mayor productividad científica ni realizó un análisis temático, ni de redes de colaboración que refleje los grupos de investigación o entidades nacionales o extranjeras que más utilicen estas encuestas, datos importantes dentro de los indicadores de producción científica.

En conclusión, el uso de los datos de estas cinco encuestas nacionales posibilitó la generación de investigaciones originales de calidad y publicadas en revistas indizadas. Se sugiere mantener la libre difusión de estos datos e impulsar su uso para la generación de investigaciones relevantes en la salud pública peruana.

Contribuciones de autoría: JCRM, AMPP y RASA han participado en la concepción y diseño del artículo, en la recolección de datos, en el análisis e interpretación de los mismos, en la redacción y revisión crítica del artículo y en la aprobación de la versión final, de manera equitativa.

Fuente de financiamiento: Autofinanciado.

Conflictos de interés: Los autores declaran no tener conflictos de interés.

\section{REFERENCIAS BIBLIOGRÁFICAS}

1. Instituto Nacional de Estadística e Informática [Internet]. Lima: Instituto Nacional de Estadística e Informática; c2017 [citado 12 septiembre 2017 ]. Historia [1 pantalla]. Disponible en: https:// proyectos.inei.gob.pe/endes/anthist.asp

2. Cheng HG, Phillips MR. Secondary analysis of existing data: opportunities and implementation. Shanghai Arch Psychiatry. 2014;26(6):371-5. doi: 10.11919/j.issn.1002-0829.214171.

3. Short Fabic M, Choi Y, Bird S. A systematic review of Demographic and Health Surveys: data availability and utilization for research. Bull World Health Organ. 2012;90(8):604-12. doi: 10.2471/BLT.11.095513.

4. Bellizzi S, Bassat Q, Ali MM, Sobel HL, Temmerman M. Effect of Puerperal Infections on Early Neonatal Mortality: A Secondary Analysis of Six Demographic and Health Surveys. PLoS One. 2017;12(1):e0170856.

5. Pernaz-Linsuy G, Cárcamo-Cavagnaro C. Conocimientos, actitudes y prácticas de la mujer peruana sobre la infección por VIH/SIDA. Rev Peru Med Exp Salud Publica. 2015;32(4):667-72.

Correspondencia: Juan Carlos Ruiz Maza

Dirección: Av. Clement 1870, Pueblo Libre. Lima, Perú.

Teléfono: (+51) 986829838

Correo electrónico:juancarlo.ruizmaza@gmail.com 